\title{
BMJ
}

\section{Duration and magnitude of the postoperative risk of venous thromboembolism in middle aged women: prospective cohort study}

\author{
Siân Sweetland, statistical epidemiologist, ' Jane Green, clinical epidemiologist, ${ }^{1}$ Bette Liu, senior research \\ fellow, ${ }^{1,4}$ Amy Berrington de González, investigator, ${ }^{1,2}$ Marianne Canonico, research associate, ${ }^{1,3}$ Gillian \\ Reeves, statistical epidemiologist, ${ }^{1}$ Valerie Beral, professor of epidemiology ${ }^{1}$ on behalf of the Million Women \\ Study collaborators
}

${ }^{1}$ Cancer Epidemiology Unit, University of Oxford, Richard Doll Building, Oxford OX3 7LF

${ }^{2}$ Division of Cancer Epidemiology and Genetics, National Cancer Institute, Bethesda, MD, USA ${ }^{3}$ Inserm Unit 780, Cardiovascular Epidemiology Section, Villejuif Cedex, France

${ }^{4}$ The George Institute for International Health, Sydney NSW, Australia

Correspondence to: V Beral pa.valerie.beral@ceu.ox.ac.uk

Cite this as: $B M J$ 2009;339:b4583 doi:10.1136/bmj.b4583

\section{ABSTRACT}

Objective To examine the duration and magnitude of increased risk of venous thromboembolism after different types of surgery.

Design Prospective cohort study (Million Women Study). Setting Questionnaire data from the Million Women Study linked with hospital admission and death records.

Participants 947454 middle aged women in the United Kingdom recruited in 1996-2001 and followed by record linkage to routinely collected NHS data on hospital admissions and deaths. During follow-up 239614 admissions were for surgery; 5419 women were admitted, and a further 270 died, from venous thromboembolism. Main outcome measures Adjusted relative risks and standardised incidence rates for hospital admission or death from venous thromboembolism (pulmonary embolism or deep vein thrombosis), by time since and type of surgery.

Results Compared with not having surgery, women were 70 times more likely to be admitted with venous thromboembolism in the first six weeks after an inpatient operation (relative risk 69.1, 95\% confidence interval 63.1 to 75.6$)$ and 10 times more likely after a day case operation $(9.6,8.0$ to 11.5$)$. The risks were lower but still substantially increased 7-12 weeks after surgery (19.6, 16.6 to 23.1 and 5.5, 4.3 to 7.0 , respectively). This pattern of risk was similar for pulmonary embolism $(n=2487)$ and deep venous thrombosis $(n=3529)$. The postoperative risks of venous thromboembolism varied considerably by surgery type, with highest relative risks after inpatient surgery for hip or knee replacement and for cancer1-6 weeks after surgery the relative risks were, respectively, 220.6 (187.8 to 259.2 ) and 91.6 (73.9 to 113.4).

Conclusion The risk of deep vein thrombosis and pulmonary embolism after surgery is substantially increased in the first 12 postoperative weeks, and varies considerably by type of surgery. An estimated 1 in 140 middle aged women undergoing inpatient surgery in the UK will be admitted with venous thromboembolism during the 12 weeks after surgery ( 1 in 45 after hip or knee replacement and 1 in 85 after surgery for cancer), compared with 1 in 815 after day case surgery and only 1 in 6200 women during a 12 week period without surgery.

\section{INTRODUCTION}

Pulmonary embolism and its precursor deep vein thrombosis (collectively termed venous thromboembolism here) are associated with a significant burden of morbidity and mortality worldwide. ${ }^{1}$ The highest risk of postoperative venous thromboembolism is during the first few weeks after surgery, ${ }^{2-4}$ but the exact pattern and magnitude of this increased risk over time is uncertain. Although the risk may vary according to indication for or type of surgery, ${ }^{5}$ with particularly high risks in patients with cancer ${ }^{6-8}$ and those undergoing major orthopaedic surgery, ${ }^{9-11}$ the magnitude and duration of risk associated with different indications for surgery is unknown. Using hospital records on admissions and death records linked with questionnaire data from a large cohort of UK women, the Million Women Study, we examined the pattern of risk of venous thromboembolism over time since surgery and by surgery type.

\section{METHODS}

The Million Women Study is a population based prospective study that recruited 1.3 million women (mean age 56) through the National Health Service (NHS) breast screening programme between 1996 and 2001 . The recruitment questionnaire (see www.million womenstudy.org) asked about various lifestyle and personal characteristics, and participants gave written consent for inclusion and follow-up.

Both England and Scotland have central databases on NHS funded hospital admissions (the hospital episode statistics ${ }^{12}$ for England and the Scottish morbidity records ${ }^{13}$ for Scotland), with individually linked data available from 1997 in England and from 1981 in Scotland. Participants in the Million Women Study were electronically linked to their inpatient and day case NHS hospital admission records in these databases 
using NHS number (a unique personal identifier used for all NHS health records), date of birth, sex, and forenames and surnames, and were similarly linked to the NHS central registers for follow-up on deaths, cancer registrations, and emigrations. ${ }^{14}$

Each hospital admission record has coded data on the primary diagnosis and up to 13 other diagnoses (coded using the ICD-10, international classification of diseases, 10th revision), as well as for up to 12 procedures (coded using the OPCS-4, Office of Population Censuses and Surveys classification of surgical operations and procedures, fourth revision ${ }^{15}$ ). The exposure of interest for these analyses was an inpatient or day case hospital admission for a surgical procedure. The main outcomes were the first diagnosis of pulmonary embolism (ICD-10 code I26) or deep vein thrombosis (I80-I82) as an inpatient or day case hospital admission (principal diagnosis or comorbidity) or as an underlying cause of death.

\section{Statistical analysis}

We excluded 207302 women from the analyses who had a hospital admission for venous thromboembolism before recruitment or reported a history of blood clot or treatment for clotting problems at recruitment, or who had previous cancer or who had an operation in the year before the start of follow-up. We excluded a further 208538 women with more than one operation during follow-up so that the risk after a single admission for surgery could be examined, thus minimising any confounding from multiple operations.

We calculated person years from the date of recruitment to whichever came first of the date of first hospital admission for venous thromboembolism, date of death (with or without a diagnosis of venous thromboembolism), date of emigration, or end of follow-up for hospital admissions, after which the hospital administration data were incomplete - for women recruited in Scotland this final date of follow-up was 31 December 2003, whereas for those recruited in England it was 31 March 2005. A small proportion of women in England (5\%) were recruited before 1 April 1997 and we calculated person years from this date for such women.

We took the person years before any operation as the reference period, which we refer to as "no surgery." This includes all person years of follow-up for women with no operations during the entire follow-up period as well as the person years before their operation for women with an operation after recruitment. We examined day case and inpatient operations separatelyinpatient operations were defined as those requiring an overnight stay in hospital and day case operations as those where women were admitted and discharged on the same day.

We used Cox regression models with attained age as the underlying time variable to estimate the relative risk of venous thromboembolism in relation to time since surgery and surgery type. These exposure variables were fitted as time dependent covariates, so that all women initially contributed person years to the reference period (no surgery), then those with an operation moved through categories representing various time periods after their surgery during follow-up. We routinely stratified analyses by area of recruitment (10 geographical regions) and socioeconomic status, defined in fifths using the Townsend method. ${ }^{16}$ Additional adjustment was made for body mass index $(<25$, $\left.25-29, \geq 30 \mathrm{~kg} / \mathrm{m}^{2}\right)$ and use of hormone replacement therapy at recruitment (never, past, current). Other potential confounders, such as use of oral contraceptives, smoking status, and alcohol consumption, and conditions such as acute myocardial infarction, heart failure, inflammatory bowel disease, respiratory failure, stroke, or varicose veins, were investigated as adjustment factors in the models, but as they had little additional effect on the relative risks estimates they were not retained as adjustment factors. We included women with missing values for any of the baseline characteristic variables in a separate category for that variable.

For the initial analyses we divided the first year after surgery into weekly periods for the first 12 weeks then monthly (in 31 day periods) up to one year, and we examined the relative risk of venous thromboembolism during each of these periods after surgery compared with the risk with no surgery. We then amalgamated these categories, based on the pattern of risk observed in our detailed analyses, into three time periods for the first postoperative year: weeks 16 , weeks $7-12$, and months $4-12$. Cancer is a known risk factor for venous thromboembolism and a common reason for surgery, so when we examined the relative risks after different types of surgery, we classified operation records with a cancer code (ICD-10 codes beginning with "C") as cancer surgery, and women without such a record were classified by OPCS-4 codes as: orthopaedic (all OPCS-4 codes beginning with "W"), vascular (all "K" and "L" codes), gynaecological (all "Q" codes), gastrointestinal (all "G" and "H" codes), and all other surgery. Because of the known association between venous thromboembolism and major orthopaedic surgery, we subdivided the orthopaedic operations into hip, knee, or femoral head replacements (specifically codes W37-W42, W46-W48), fracture surgery (codes W19-W26), and all other types of orthopaedic surgery (remaining "W" codes). For the few cases $(<0.5 \%$ of all operation episodes) with more than one operation code on the same hospital record, we applied a hierarchy in the following order for classifying the overall operation: cancer, hip or knee replacement, fracture, other orthopaedic operations, and vascular, gynaecological, gastrointestinal, and all other surgery.

We calculated standardised incidence rates for hospital admission or death from venous thromboembolism using incidence rates without surgery as the standard and standardising for region, socioeconomic group, body mass index, and use of hormone replacement therapy. Relative risks are reported with 95\% confidence intervals; all analyses were done using Stata 10.1 software. 


\begin{tabular}{|c|c|c|c|}
\hline \multirow[b]{2}{*}{ Characteristics } & \multicolumn{2}{|c|}{ Operation during follow-up } & \multirow[b]{2}{*}{ All women } \\
\hline & No & Yes & \\
\hline No of women & 707840 & 239614 & 947454 \\
\hline \multicolumn{4}{|l|}{ Baseline: } \\
\hline Mean (SD) age (years) & $55.8(4.6)$ & $56.1(4.7)$ & $55.8(4.6)$ \\
\hline Mean (SD) body mass index (weight $(\mathrm{kg}) /\left(\right.$ height) $\mathrm{m}^{2}$ ) & $25.9(4.5)$ & $26.3(4.7)$ & $26.0(4.6)$ \\
\hline Current user of hormone replacement therapy & 31.2 & 35.9 & 32.4 \\
\hline Ever user of oral contraceptives & 59.6 & 58.9 & 59.4 \\
\hline Mean (SD) alcohol intake among drinkers (g/day) & $8.4(7.8)$ & $8.0(7.6)$ & $8.3(7.8)$ \\
\hline Lowest socioeconomic fifth & 18.1 & 20.2 & 18.6 \\
\hline Strenuous physical activity more than once a week & 22.2 & 20.5 & 21.8 \\
\hline Postmenopausal & 84.6 & 85.9 & 84.9 \\
\hline Mean (SD) No of children & $2.1(1.2)$ & $2.2(1.2)$ & $2.1(1.2)$ \\
\hline Current smoker & 19.8 & 20.5 & 20.0 \\
\hline \multicolumn{4}{|l|}{ Follow-up: } \\
\hline Person years of follow-up & 4343876 & 1491458 & 5835334 \\
\hline No (\%) of women with incident venous thromboembolism during follow-up & $3589(0.5)$ & $2100(0.9)$ & $5689(0.6)$ \\
\hline
\end{tabular}

\section{RESULTS}

Overall, 947454 women were included in the main analyses, and participants were followed for a total of 5.84 million person years (mean 6.2 years per woman), during which time 239614 women $(25 \%)$ had an operation (149 355 as a day case and 90259 as an inpatient). Table 1 shows characteristics of the study population according to whether or not they had an operation during follow-up. The two groups were broadly similar for baseline characteristics, except that women who had an operation after recruitment were slightly more likely to have been current users of hormone replacement therapy.

During follow-up 5419 women were admitted to hospital with pulmonary embolism or deep vein thrombosis and 270 had a first diagnosis of venous thromboembolism at death. These 5689 women $(0.6 \%$ of the study population) are described here as having a first venous thromboembolic event, regardless of whether it was a hospital admission or a death.

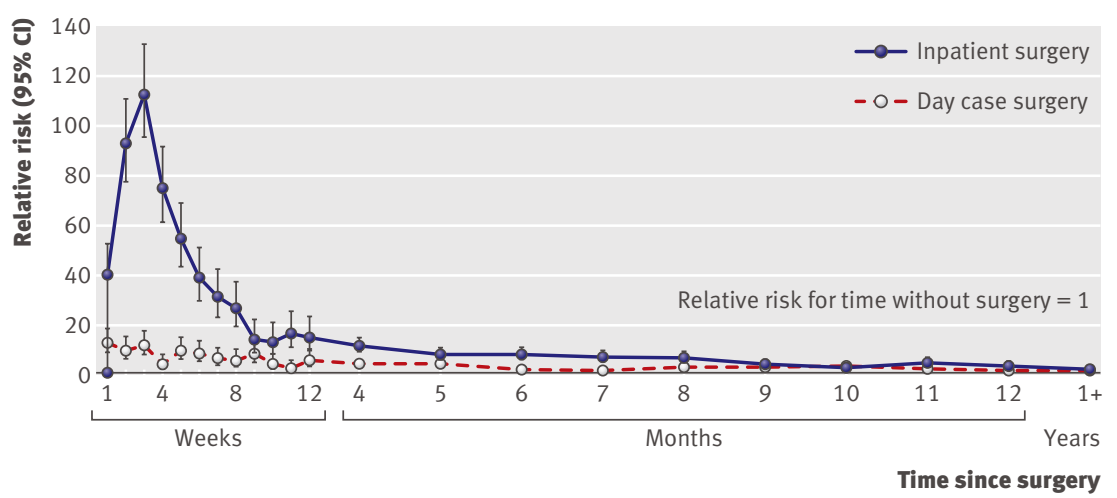

Fig 1| Relative risk of venous thromboembolism by time since inpatient surgery and since day case surgery
Table 2 shows the distribution of venous thromboembolism events by time since surgery. More than one third of all such events in this population were diagnosed among the $25 \%$ of women who had surgery, with $16 \%$ of all cases diagnosed in the 12 weeks after surgery ( $3 \%$ after day case surgery, $13 \%$ after inpatient surgery). In contrast, only $1 \%$ of the total person years occurred in the 12 weeks after surgery.

\section{Relative risks over time since surgery}

The relative risk of venous thromboembolism after surgery varied considerably by time since surgery, particularly after an inpatient operation (fig 1). Compared with incidence rates without surgery, women were 40 times more likely to develop venous thromboembolism during the week after an inpatient operation (relative risk $40.3,30.7$ to 52.8$)$, rising to 110 times more likely during the third postoperative week (112.5, 95.3 to 132.8$)$. The risk then decreased, but remained substantially elevated up to 12 weeks postoperatively. The overall relative risk was 69.1 (63.1 to 75.6) for the first six weeks after surgery, 19.6 (16.6 to 23.1) 7-12 weeks postoperatively, 9.4 (8.0 to 11.1) 4-6 months postoperatively, 6.1 (5.0 to 7.5) 7-9 months postoperatively, and 3.7 (2.8 to 4.9 ) 10-12 months postoperatively.

After day case surgery the relative risks of venous thromboembolism were lower than after inpatient surgery but still significantly increased, at almost 10 for the first six weeks after surgery $(9.6,8.0$ to 11.5$)$ and about six for $7-12$ weeks after surgery $(5.5,4.3$ to 7.0$)$. The relative risks after day case surgery were still slightly increased afterwards, at 3.7 (3.0 to 4.5) 4-6 months after surgery, 2.6 (2.0 to 3.3) 7-9 months after surgery, and 2.6 (2.0 to 3.4) 10-12 months after surgery.

The overall patterns of relative risk were similar in analyses taking pulmonary embolism and deep vein thrombosis as separate outcomes (fig 2). 


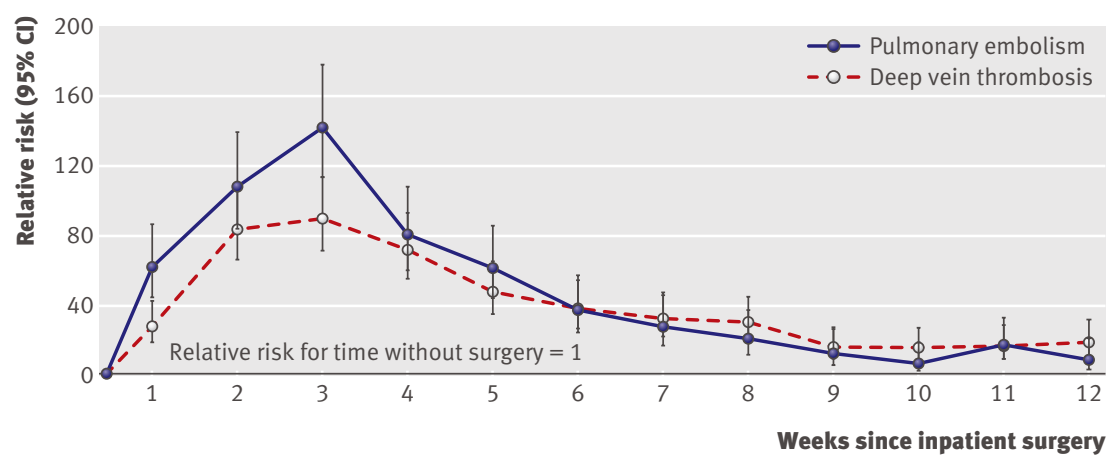

Fig 2 2 Relative risks of pulmonary embolism and deep vein thrombosis by time since inpatient surgery replacement and fracture surgery decreased rapidly from those in the first six weeks to: 39.7 (27.3 to 57.8) and 39.8 (25.0 to 63.4), respectively. The relative risks after inpatient surgery for vascular, gastrointestinal, and gynaecological conditions were all increased more than 10-fold (15.8 (8.5 to 29.3), 18.5 (9.9 to 34.6 ), and 11.1 (5.9 to 20.6), respectively), and the relative risk after other orthopaedic surgery and all other inpatient surgery were still more than five times that without surgery $(5.6,2.1$ to 14.9 and $8.4,5.6$ to 12.6 , respectively).

The increased relative risk associated with inpatient surgery for cancer was still evident 4-12 months after surgery $(34.4,29.3$ to 40.2$)$ and one year or more after surgery (6.1, 4.9 to 7.6). The overall relative risk after inpatient surgery for non-cancer conditions was 3.3 (2.8 to 3.9) 4-12 months after surgery and 1.7 (1.5 to $1.9)$ one year or more after surgery.

Few operations for joint replacement or fracture were done as day cases so the risks associated with these procedures could not be estimated. Cancer dominated the findings for day case surgery (fig 4), with relative risks of 80.4 (54.0 to 119.6 ) for the six weeks after surgery, 47.7 (28.6 to 79.6$)$ for the 7-12 weeks after surgery, and 12.1 (7.9 to 18.7) for the 4-12 months after surgery. Relative risks were more than 20 during the first six weeks after day case vascular surgery and other orthopaedic surgery $(26.4,17.2$ to 40.6 and $22.9,14.4$ to 36.5 ), and more than doubled after other types of day case surgery: 2.5 (1.0 to 6.8 ) after gynaecological surgery, 5.3 (3.5 to 8.1) after gastrointestinal surgery, and 6.2 (4.3 to 8.9$)$ after other surgery.

Results for the separate outcomes of pulmonary embolism and deep vein thrombosis showed similar overall patterns in relation to operation type to the main venous thromboembolism analyses, although confidence intervals were wider owing to smaller numbers in each category (data not shown).

\section{Event rates}

The incidence rates are calculated using both hospital admissions and death records for venous thromboembolism. The incidence rate for venous thromboembolism during all time without surgery was 0.7 per

Table 2|Person years and number of women with incident venous thromboembolism, by time since surgery. Values are numbers (percentages) unless stated otherwise

\begin{tabular}{|c|c|c|c|c|}
\hline \multirow[b]{2}{*}{ Time since surgery } & \multirow[b]{2}{*}{ Person years $(\%)$} & \multicolumn{3}{|c|}{ Diagnosis on hospital admission or death record } \\
\hline & & $\begin{array}{l}\text { Deep vein thrombosis or } \\
\text { pulmonary embolism }\end{array}$ & Deep vein thrombosis $†$ & Pulmonary embolism $\ddagger$ \\
\hline No surgery & $5136506(88)$ & $3589(63)$ & $2248(64)$ & $1547(62)$ \\
\hline 1-6 weeks after surgery & $27187(0.5)$ & $675(12)$ & $377(11)$ & $340(14)$ \\
\hline 7-12 weeks after surgery & $26363(0.5)$ & $218(4)$ & $145(4)$ & $86(3)$ \\
\hline 4-12 months after surgery & $159153(3)$ & $520(9)$ & $337(10)$ & $210(8)$ \\
\hline$\geq 1$ year after surgery & $486126(8)$ & $687(12)$ & $422(12)$ & $304(12)$ \\
\hline \multicolumn{5}{|c|}{$\begin{array}{l}\text { Numbers do not add up to total number of women with venous thromboembolism, and corresponding person years also varies slightly because } \\
\text { women are censored at different times in each analysis and some women have had admissions for both conditions. } \\
\text { *Women with pulmonary embolism or deep vein thrombosis. } \\
\text { †Women with pulmonary embolism. } \\
\text { †Women with deep vein thrombosis. }\end{array}$} \\
\hline
\end{tabular}


1000 person years, which is equivalent to 0.058 per 1000 person months. Corresponding standardised incidence rates were 2.6 per 1000 person months in the 12 weeks after inpatient surgery, 0.4 per 1000 person months in the 4-12 months after inpatient surgery, and 0.1 per 1000 person months after one year or more, compared with $0.4,0.2$, and 0.09 per 1000 person months, respectively, after day case surgery. These higher rates in the 12 weeks after surgery varied considerably by indication for surgery or surgery type (table 3): after inpatient surgery the highest rates were 4.3 per 1000 person months for cancer, 7.7 per 1000 person months for hip or knee replacement, and

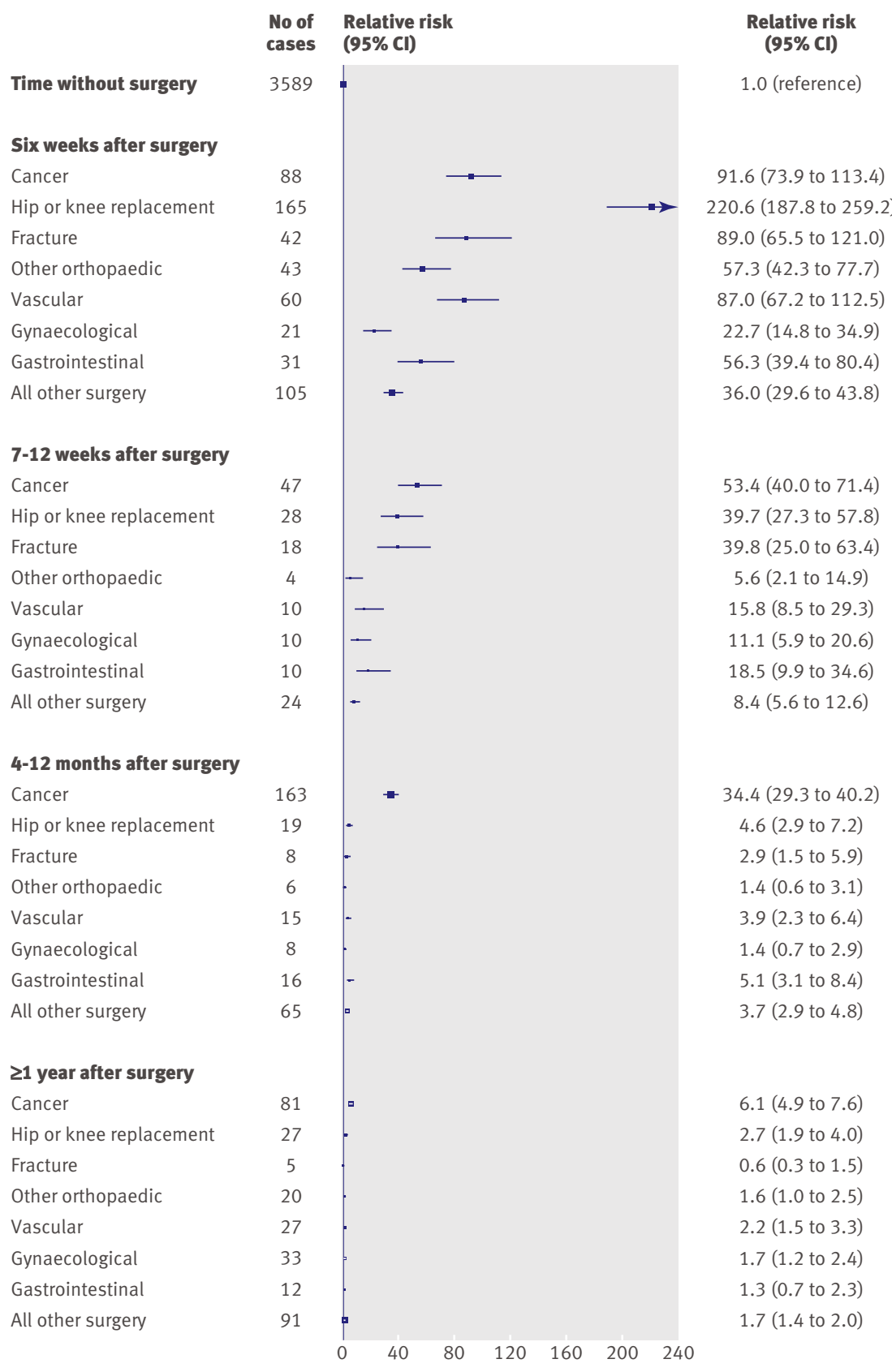

Fig 3 | Relative risk of venous thromboembolism by time since inpatient surgery and by surgery type. Relative risks are adjusted by age, region, socioeconomic status, body mass index, and use of hormone replacement therapy
3.8 per 1000 person months for fracture surgery, followed by 3.1 per 1000 person months for vascular surgery and 2.2 per 1000 person months for gastrointestinal surgery, whereas all other types of non-cancer inpatient surgery were associated with rates of less than 2 per 1000 person months; after day case surgery the rate for cancer surgery was 3.7 per 1000 person months, and for other types of surgery was less than 1 per 1000 person months.

Over a 12 week period without surgery about 1 in 6200 middle aged women in the UK will be admitted to hospital for, or die from, venous thromboembolism compared with 1 in 815 during the 12 weeks after a day case operation, and 1 in 140 women after an inpatient operation.

\section{DISCUSSION}

In this large prospective study of middle aged women in the UK, the risk of hospital admission for, or death from, venous thromboembolism varied considerably during the year after an operation. The risk was highest during the first six weeks after inpatient surgery, reaching a peak in the third postoperative week. Incidence rates for venous thromboembolism in the first six postoperative weeks were over 100 times the rates without surgery, and 7-12 weeks after inpatient surgery rates were still almost 20 times higher than without surgery. The relative risks after day case surgery were lower than for inpatient surgery but still substantially increased. The risk of postoperative venous thromboembolism also varied by type of surgery, being highest in the six weeks after hip or knee replacement surgery and in the six weeks after surgery for cancer.

These findings suggest that the risk of venous thromboembolism after surgery is greater and lasts for longer than previously thought. To our knowledge no other study has looked at the risks of venous thromboembolism in such fine time periods after different types of surgery. One previous report suggested that the risk of venous thromboembolism in the six months after surgery was nine times higher than without surgery, ${ }^{4}$ another reported a 22-fold increased risk in the 90 days after surgery, ${ }^{17}$ and another study reported that the risk of pulmonary embolism was increased in the second postoperative week, returning to a similar risk to the first postoperative week one month after surgery. ${ }^{2}$ Although one study ${ }^{4}$ reported a small (10\%) increased risk of venous thromboembolism six or more months after surgery, most studies simply reported the mean or median time to venous thromboembolism after major surgery, generally after orthopaedic surgery. ${ }^{1819}$ Overall, we found increased relative risks of venous thromboembolism during the 12 postoperative weeks that were much larger than those reported previously.

The recent National Institute for Health and Clinical Excellence (NICE) guidelines for the prevention of venous thromboembolism specified inpatient surgery $^{9}$ only. Few studies have directly compared the risks of venous thromboembolism after day case and inpatient surgery, ${ }^{20}$ and although some examined 


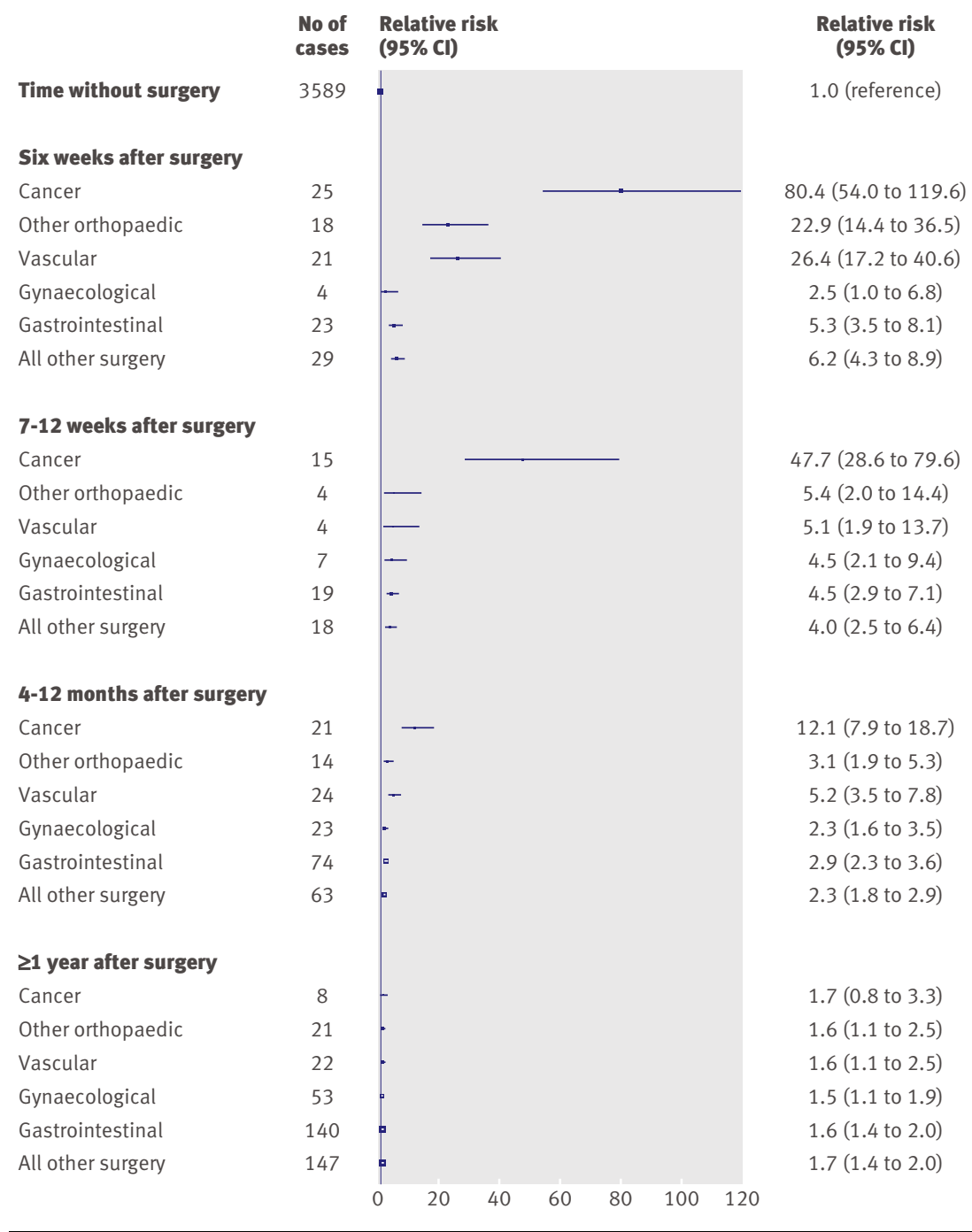

Fig 4 | Relative risk of venous thromboembolism by time since day case surgery and by surgery type. Relative risks are adjusted by age, region, socioeconomic status, body mass index, and use of hormone replacement therapy. *Joint replacements after day case fracture surgery were insufficient to estimate relative risks

the risk after day surgery alone, ${ }^{2122}$ most analyses were restricted to events occurring within the first two months. Although we found that the risks of venous thromboembolism after inpatient surgery were much higher than after day case surgery, the risks were still eight times higher in the 12 weeks after a day case operation than without surgery.

Major orthopaedic surgery - generally of the lower limb (hip and knee replacements and surgery for hip fracture) where deep vein thromboses tend to occurhas been associated with very high risks of postoperative venous thromboembolism. ${ }^{2181923}$ Most women undergoing hip or knee replacement or fracture surgery were admitted as inpatients, and we found that during the first six weeks after these operations the risks were increased some 200 -fold and 90-fold, respectively, and other inpatient orthopaedic operations were increased about 60 -fold. The relative risks after inpatient surgery for hip or knee replacement and fracture were still about 40 in the 7-12 weeks after surgery. In the recent NICE guidelines for prevention of venous thromboembolism in surgical inpatients, ${ }^{9}$ the recommendation for extended drug prophylaxis (for up to four weeks after surgery) for inpatients after surgery for hip fracture, or hip replacement was only if there was at least one other predisposing factor. Similarly, the Scottish guidelines ${ }^{10}$ (from 2002) recommend drug prophylaxis extending to four or five weeks after orthopaedic surgery only for high risk patients after hip or knee replacement. Although women undergoing major orthopaedic surgery are more likely than others to have been given some thromboprophylaxis after hospital discharge, the very high incidence of venous thromboembolism after orthopaedic operations remains.

The strong association between cancer and venous thromboembolism is known, ${ }^{6824}$ and the relative risks associated with cancer surgery were substantially higher than those associated with most other indications for surgery. The increased relative risk that persisted for a year or longer found here may be due to the underlying cancer itself rather than to a longlasting effect of the surgery itself.

\section{Strengths and limitations of the study}

We excluded from the analyses all women who reported being treated for clotting problems, but we had no information on perioperative prophylactic measures, such as compression stockings or drugs. The use of such prophylactic measures would be expected to vary by type of surgery and possibly also between hospitals. The characteristics of the patient, including coexisting risk factors for venous thromboembolism, are also likely to influence the prophylactic measures given, and the prophylaxis itself would be expected to affect the timing of any postoperative venous thromboembolism. Some of these factors such as body mass index and use of hormone replacement therapy have been taken into account by adjustments for baseline characteristics, but information on other risk factors, such as immobility, is lacking. Although we have no way of knowing exactly what regimens of prophylaxis these women received, there are many national and international guidelines for the prevention of venous thromboembolism ${ }^{9-11}$ and it is likely that most women undergoing major surgery received some form of prophylaxis, ${ }^{25-27}$ at least until hospital discharge. Those undergoing procedures within a day case admission were probably less likely to receive prophylaxis. $\mathrm{NICE}^{9}$ suggests mechanical prophylaxis (compression stockings) for all inpatients having surgery, plus the offer of low molecular weight heparin (or fondaparinux) for those with an additional risk factor and those undergoing major orthopaedic surgery, when prophylaxis may be considered for up to four weeks. Consequently, the magnitude of the increased relative risks of venous thromboembolism after inpatient surgery seen here might have been higher still in the absence of prophylaxis, particularly during the first few postoperative weeks.

Since we classified any operative procedure as surgery here ${ }^{28}$ minor procedures, such as biopsies, were 
Table 3 |Estimated incidence rates of hospital admission or death for venous thromboembolism among middle aged women (50-64 years)

\begin{tabular}{|c|c|c|c|c|}
\hline \multirow[b]{2}{*}{$\begin{array}{l}\text { Indication for surgery and } \\
\text { type of surgery }\end{array}$} & \multicolumn{2}{|c|}{ Inpatient surgery } & \multicolumn{2}{|c|}{ Day case surgery } \\
\hline & $\begin{array}{l}\text { Incidence rate per } 1000 \\
\text { person months }\end{array}$ & $\begin{array}{c}\text { No who develop VTE over } \\
12 \text { weeks }\end{array}$ & $\begin{array}{l}\text { Incidence rate per } 1000 \\
\text { person months }\end{array}$ & $\begin{array}{c}\text { No who develop VTE over } \\
12 \text { weeks }\end{array}$ \\
\hline No surgery & $0.058^{\star}$ & 1 in 6200 & $0.058^{\star}$ & 1 in 6200 \\
\hline After surgery†: & 2.61 & 1 in 140 & 0.44 & 1 in 815 \\
\hline Cancer surgery & 4.27 & 1 in 85 & 3.73 & 1 in 95 \\
\hline Hip or knee replacement & 7.74 & 1 in 45 & Insufficient data & Insufficient data \\
\hline Fracture & 3.78 & 1 in 95 & Insufficient data & Insufficient data \\
\hline Other orthopaedic surgery & 1.87 & 1 in 195 & 0.84 & 1 in 430 \\
\hline Vascular surgery & 3.07 & 1 in 115 & 0.93 & 1 in 390 \\
\hline Gynaecological surgery & 0.99 & 1 in 365 & 0.20 & 1 in 1765 \\
\hline Gastrointestinal surgery & 2.19 & 1 in 165 & 0.29 & 1 in 1255 \\
\hline Other surgery & 1.30 & 1 in 275 & 0.30 & 1 in 1210 \\
\hline
\end{tabular}

VTE=venous thromboembolism.

*Equal to 0.70 per 1000 person years.

tIncidence rates are averages over the first 12 postoperative weeks.

included. To avoid any effect of multiple operations on our estimates of the risk of venous thromboembolism after surgery, we excluded all women with more than one operation during follow-up. The risks associated with repeated operations may well be different from those reported here, particularly as those with multiple operations may be more likely to have increased immobility and other factors affecting their risk of venous thromboembolism owing to the severity of their underlying conditions.

Hospital admissions for venous thromboembolism occurring outside of the NHS in England and Scotland would not be included in this dataset; however, privately funded admissions are relatively rare in the $\mathrm{UK}^{2930}$ and we have previously shown good agreement between self reported operations and NHS hospital admission data. ${ }^{31}$ In addition, if the index operation was carried out in an NHS hospital, it is likely that any subsequent admission for venous thromboembolism would also be in an NHS hospital; consequently it is unlikely that many postoperative venous thromboembolic events have been missed.

Although the diagnosis of pulmonary embolism can be difficult, ${ }^{32}$ the severity of this condition means that most cases will be treated as an inpatient or day case hospital admission (or result in death). Therefore symptomatic or fatal pulmonary embolism should

\section{WHAT IS ALREADY KNOWN ON THIS TOPIC}

It is well known that the risk of venous thromboembolism is increased after surgery

Information on how long this risk lasts or how the risk varies by type of surgery is, however, limited

\section{WHAT THIS STUDY ADDS}

The postoperative risk of venous thromboembolism reaches a peak about three weeks after surgery, but risks are substantially increased up to 12 weeks postoperatively

Risks are much greater after inpatient surgery than after day case surgery but are still significantly elevated after day case surgery

Risks are greatest after joint replacement and surgery for cancer rarely go undetected in this study population. In contrast, increasing numbers of cases of deep vein thromboses occurring in the general population are treated in a community setting (general practice) or in outpatient setting, leading to underestimation of the total incidence of deep vein thrombosis after surgery if based on hospital admissions alone. Also, women with deep vein thrombosis may be more likely to be admitted as an inpatient or day case if they have other predisposing factors, including recent surgery or certain medical conditions, such as cancer. The patterns of risk after surgery were, however, similar for pulmonary embolism and for deep vein thrombosis when each was examined separately (fig 2). Because we have no information on non-fatal venous thromboembolism (mainly deep vein thrombosis) occurring outside of the inpatient or day case hospital admissions, our estimates of incidence rates of venous thromboembolism are for inpatient and day case admissions only.

This study has several strengths. Although venous thromboembolism is relatively rare in the general population $(0.6 \%$ of these participants in the Million Women Study experienced a venous thromboembolism event during an average of 6.2 person years of follow-up), the large sample size allowed a detailed investigation into the magnitude and duration of increased risk over time since surgery. Access to information on a wide range of potential confounders was used as adjustment factors. The accuracy of the linkage process and the recording of operations and pulmonary embolism have also been shown to be good. ${ }^{132831}$ The prospective design of the study, with record linkage to NHS hospital admissions and cancer and death records, virtually complete follow-up, and objective recording both of operations and of venous thromboembolism, should provide reliable estimates of risk.

\section{Implications for practice}

The incidence of venous thromboembolism after surgery is high for middle aged women. Recommendations for thromboprophylaxis vary, and the recent 
NICE guidelines ${ }^{9}$ suggest extended duration of drug prophylaxis (that is, beyond hospital discharge) after hip replacement and surgery for hip fracture only, when it is recommended for up to four weeks. The findings from this study suggest that there is a substantially increased risk of venous thromboembolism after many different types of surgery that lasts for up to 12 weeks postoperatively.

We thank the women who participated in the Million Women Study; the study steering committee as well as ISD Scotland, the Information Centre for Health and Social Care, and Northgate Solutions for the linkage of hospital records; and Adrian Goodill for his assistance in the preparation of the figures. A Cancer Research UK studentship supported SS for the duration of her DPhil studies, which included some of the work in this paper. Million Women Study steering committee: Joan Austoker, Emily Banks, Valerie Beral, Judith Church, Ruth English, Jane Green, Julietta Patnick, Richard Peto, Gillian Reeves, Martin Vessey, and Matthew Wallis. Million Women Study coordinating centre staff: Simon Abbott, Miranda Armstrong, Krys Baker, Angela Balkwill, Vicky Benson, Valerie Beral, Judith Black, Anna Brown, Diana Bull, Benjamin Cairns, James Chivenga, Barbara Crossley, Dave Ewart, Sarah Ewart, Lee Fletcher, Laura Gerrard, Adrian Goodill, Isobel Green, Jane Green, Elizabeth Hilton, Joy Hooley, Sau Wan Kan, Carol Keene, Oksana Kirichek, Nicky Langston, Bette Liu, Maria-Jose Luque, Maria MacGregor, Lynn Pank, Kirstin Pirie, Gillian Reeves, Emma Sherman, Evie Sherry-Starmer, Moya Simmonds, Elizabeth Spencer, Helena Strange, Siân Sweetland, Alison Timadjer, Sarah Tipper, Ruth Travis, Xiaosi Wang, Joanna Watson, Stephen Williams, Lucy Wright, Tienyu Yang, and Heather Young. Collaborating UK NHS Breast Screening Centres: Avon, Aylesbury, Barnsley, Basingstoke, Bedfordshire and Hertfordshire, Cambridge and Huntingdon, Chelmsford and Colchester, Chester, Cornwall, Crewe, Cumbria, Doncaster, Dorset, East Berkshire, East Cheshire, East Devon, East of Scotland, East Suffolk, East Sussex, Gateshead, Gloucestershire, Great Yarmouth, Hereford and Worcester, Kent, Kings Lynn, Leicestershire, Liverpool, Manchester, Milton Keynes, Newcastle, North Birmingham, North East Scotland, North Lancashire, North Middlesex, North Nottingham, North of Scotland, North Tees, North Yorkshire, Nottingham, Oxford, Portsmouth, Rotherham, Sheffield, Shropshire, Somerset, South Birmingham, South East Scotland, South East Staffordshire, South Derbyshire, South Essex, South Lancashire, South West Scotland, Surrey, Warrington Halton St Helens and Knowsley, Warwickshire Solihull and Coventry, West Berkshire, West Devon, West London, West Suffolk, West Sussex, Wiltshire, Winchester, Wirral, and Wycombe.

Contributors: All authors contributed to the design and conduct of the study and read and approved the final manuscript. VB and SS are the guarantors.

Funding: This study was funded by Cancer Research UK (grant No C570/ A5028) and the Medical Research Council (grant No G0700474). Marianne Canonico was additionally supported by Leem Recherche. The funders did not participate in the study design and conduct, in the collection, management, analysis and interpretation of the data, nor was there any funder input into the preparation, review or approval of this manuscript.

Competing interests: None declared.

Ethical approval: This study was approved by the Oxford and Anglia multicentre research and ethics committee.

Data sharing: All information provided is stored in accordance with the Data Protection Act (Office of Data Protection Registrar registration No K3039784). Only the study team has access to computerised data, via passwords (see the Million Women Study protocol at www. millionwomenstudy.org).

1 House of Commons Health Committee. The prevention of venous thromboembolism in hospitalised patients. London: Stationery Office, 2005.

2 Johnson R, Green JR, Charnley J. Pulmonary embolism and its prophylaxis following the Charnley total hip replacement. Clin Orthop Relat Res 1977:123-32.

3 Huber O, Bounameaux H, Borst F, Rohner A. Postoperative pulmonary embolism after hospital discharge. An underestimated risk. Arch Surg 1992;127:310-3.

4 Huerta C, Johansson S, Wallander MA, Garcia Rodriguez LA. Risk factors and short-term mortality of venous thromboembolism diagnosed in the primary care setting in the United Kingdom. Arch Intern Med 2007;167:935-43.

5 Kearon C. Duration of venous thromboembolism prophylaxis after surgery. Chest 2003;124(suppl 6):S386-92.
6 Prandoni P, Falanga A, Piccioli A. Cancer and venous thromboembolism. Lancet Oncol 2005;6:401-10.

7 Bergqvist D. Risk of venous thromboembolism in patients undergoing cancer surgery and options for thromboprophylaxis. J Surg Oncol 2007;95:167-74.

8 Heit JA. Cancer and venous thromboembolism: scope of the problem. Cancer Control 2005;12(suppl 1):5-10.

9 National Institute for Health and Clincial Excellence. Reducing the risk of venous thromboembolism (deep vein thrombosis and pulmonary embolism) in inpatients undergoing surgery. London: NICE, 2007.

10 Scottish Intercollegiate Guidelines Network. Prophylaxis of venous thromboembolism - a national clinical guideline. Edinburgh: SIGN, 2002.

11 Geerts WH, Bergqvist D, Pineo GF, Heit IA, Samama CM, Lassen MR, et al. Prevention of venous thromboembolism: American College of Chest Physicians Evidence-Based Clinical Practice Guidelines, 8th ed. Chest 2008;133(suppl 6):S381-453.

12 NHS Information Centre. HESonline-Hospital Episode Statistics. 2008. www.hesonline.nhs.uk.

13 Kendrick SW, Clarke J. The Scottish record linkage system. Health Bull 1993;51:72-9.

14 Million Women Study collaborative group. The Million Women Study: design and characteristics of the study population. Breast Cancer Res 1999;1:73-80.

15 Office of Population Censuses and Surveys. Tabular list of the classification of surgical operations and procedures, 4th revision. London: HMSO, 1990.

16 Townsend $\mathrm{P}$, Phillimore $\mathrm{P}$, Beattie $\mathrm{A}$. Health and deprivation: inequality and the North. London: Croom Helm, 1988.

17 Heit JA, Silverstein MD, Mohr DN, Petterson TM, O’Fallon WM, Melton LJ, 3rd. Risk factors for deep vein thrombosis and pulmonar embolism: a population-based case-control study. Arch Intern Med 2000;160:809-15.

18 Warwick D, Friedman RJ, Agnelli G, Gil-Garay E, Johnson K, FitzGerald G, et al. Insufficient duration of venous thromboembolism prophylaxis after total hip or knee replacement when compared with the time course of thromboembolic events: findings from the Global Orthopaedic Registry. J Bone Joint Surg Br 2007;89:799-807.

19 Bjornara BT, Gudmundsen TE, Dahl OE. Frequency and timing of clinical venous thromboembolism after major joint surgery. J Bone Joint Surg Br 2006;88:386-91.

20 Howie $\mathrm{C}$, Hughes $\mathrm{H}$, Watts $\mathrm{AC}$ Venous thromboembolism associated with hip and knee replacement over a ten-year period: a populationbased study. J Bone Joint Surg Br 2005;87:1675-80.

21 Hoppener MR, Ettema HB, Kraaijenhagen RA, Verheyen CC, Henny PC. Day-care or short-stay surgery and venous thromboembolism. J Thromb Haemost 2003;1:863-5.

22 Ahonen J. Day surgery and thromboembolic complications: time for structured assessment and prophylaxis. Curr Opin Anaesthesiol 2007;20:535-9.

23 White RH, Romano PS, Zhou H, Rodrigo J, Bargar W. Incidence and time course of thromboembolic outcomes following total hip or knee arthroplasty. Arch Intern Med 1998;158:1525-31.

24 Lee AY. Cancer and venous thromboembolism: prevention, treatment and survival. / Thromb Thrombolysis 2008;25:33-6.

25 McEleny P, Bowie P, Robins JB, Brown RC. Getting a validated guideline into local practice: implementation and audit of the SIGN guideline on the prevention of deep vein thrombosis in a district general hospital. Scott Med J 1998;43:23-5.

26 Walker A, Campbell S, Grimshaw J. Implementation of a national guideline on prophylaxis of venous thromboembolism: a survey of acute services in Scotland. Thromboembolism Prevention Evaluation Study Group. Health Bull (Edinb) 1999;57:141-7.

27 Cohen AT, Tapson VF, Bergmann JF, Goldhaber SZ, Kakkar AK, Deslandes B, et al. Venous thromboembolism risk and prophylaxis in the acute hospital care setting (ENDORSE study): a multinational cross-sectional study. Lancet 2008;371:387-94.

28 Information Services Division. An assessment of SMR01 and associated data 2004-2006. Edinburgh: ISD Scotland, 2007.

29 NHS Scotland Resource Allocation Committee. Private health sector services and resource allocation in Scotland. Edinburgh: Scottish Executive Health Department, 2005.

30 Williams B, Whatmough P, McGill J, Rushton L. Patients and procedures in short-stay independent hospitals in England and Wales, 1997-1998. J Public Health Med 2000;22:68-73.

31 Liu B, Sweetland S, Beral V, Green J, Balkwill A, Casabonne D. Selfreported information on joint replacement and cholecystectomy agrees well with that in medical records. J Clin Epidemiol 2007;60:1190-4.

32 British Thoracic Society guidelines for the management of suspected acute pulmonary embolism. Thorax 2003;58:470-83.

Accepted: 14 September 2009 